\title{
Numerical Study on Influence of Canard on Static Aeroelastic Characteristics of Forward-Swept Wing
}

\author{
Binlin Ma ${ }^{a}$, Xinbing $\mathrm{Su}^{\mathrm{b}}$, Taorui $\mathrm{Li}^{\mathrm{c}}$ and Ning Wang ${ }^{\mathrm{d}}$ \\ Aeronautics and Astronautics Engineering College of Air Force Engineering University, Xi'an \\ 710038, China \\ ambinlin001@163.com, bsxinbing@sohu.com, c15596652671@163.com, d1289603317@qq.com
}

Keywords: Forward-swept wing, Canard, Static aeroelasticity, CFD/CSD.

\begin{abstract}
The research on configuration of canard with forward-swept wing is of great significance in modern fighters' design. According to the influence of canard on static aeroelasticity of forward-swept wing, the longitudinal aerodynamic and elastic characteristics of canard with forward-swept wing configuration was calculated based on computational fluid dynamics/computational structural dynamics (CFD/CSD) numerical method, then compared with forward-swept wing configuration. It's resulted that is the critical value, below which the down-wash flow of canard is bad for wing or else good by vortex of canard controlling the separation of wing. The bending and torsion deformation of forward-swept wing is suppressed under the influence of the canard at middle angle of attack, which benefits for improving static aeroelastic stability. The conclusion also can provide references for the design of fighters with forward-swept configuration.
\end{abstract}

\section{Introduction}

It is of great significance to study the canard with forward-swept wing in fighter design. Because of the better stall characteristic and the lower transonic resistance, the forward-swept wing aircraft has the potential superiority in high attack angle. The down wash of canard effectively delay the separation in the root of forward-swept wing and has favorable interference on the flow of forward-swept wing [1].

Experiments and simulations have been carried out to study the effects of canard on aerodynamic characteristics of forward-swept wing. It's researched [2-4] that the forward-swept wing has obvious aerodynamic advantage. The canard has important effects on the aerodynamic characteristics of the forward-swept wing and reasonable position of canard can further increase the aerodynamic advantage of the forward-swept wing. The structure of modern fighters tends to improve flexibility, as the result of which aeroelasticity is more prominent [5].However, the forward-swept wing has the shortcomings of static and dynamic elastic divergence, so it is necessary to study the effects of canard on static aeroelastic characteristics of forward-swept wing.

In recent years, numerical simulation has gradually become the main method to study static aerodynamic characteristics of aircraft [6]. In this paper, the calculation and analysis of static aeroelastic characteristics of the rigid canard coupling elastic forward-swept wing are performed based on the improved computational fluid dynamics/computational structural dynamics (CFD / CSD) loosely coupled numerical method [7].

\section{Numerical method and Calculating model}

The CFD / CSD loosely coupled numerical simulation method is the main method of static aeroelastic analysis. The fluid controlling equations and the structural static equations are coupled to solve, but the model of fluid and structure are separately solved in the CFD and CSD solvers. In each iteration, the aerodynamics obtained by the CFD solver is transmitted to the structural grid, and the structural deformation displacement solved from the CSD solver is transmitted to the flow field grid. Before the next iteration carried out, the update of flow field grid is performed through using the 
dynamic grid technique. Until aerodynamic and structural displacements are convergent, the above process is operated repeatedly.

\subsection{Flow model}

Aerodynamic solution technology based on CFD is the key to CFD / CSD coupling calculation, and the three-dimensional Reynolds-averaged Navier-Stokes (RANS) equation is used to calculate aerodynamics. The integral form of the conservation of three-dimensional N-S equations can be written as follows:

$$
\frac{\partial}{\partial t} \iiint_{\Omega} \mathbf{Q} d V+\iint_{S}\left(\mathbf{G}-\mathbf{F}^{v}\right) \cdot \mathbf{n} d S=0
$$

Where: $\mathbf{Q}, \mathbf{G}$ and $\mathbf{F}^{v}$ are the solution vector, inviscid flux vectors and viscous flux vectors, respectively. Control volume and surface of control volume are respected by $\Omega$ and $S$ respectively. And $\mathbf{n}$ respects the surface normal.

\subsection{Structural static equations}

It is difficult to solve the deformation of complex structures under loading by using structural mechanics method. Therefore, the finite element method is used to disperse the structures into finite small units. After the structural model is discretized, the structural deformation can be obtained by solving the static equilibrium equation:

$$
\mathbf{K}\{q\}=\mathbf{F}
$$

Where $\mathbf{K}$ is the structural stiffness matrix, $\{q\}$ is the displacement vector of the node, and $\mathbf{F}$ is the load vector acting on the node.

\subsection{Data transmission and mesh deformation}

When the aero-elastic problem is solved in the time domain by loosely-coupled method, the data exchange between the aerodynamic and structural mesh needs to be done by interpolation in each time step. The interpolation of the displacement and load information is carried out by using the modified constant volume conversion (CVT) method [8]. In static aeroelastic calculation, the deformation of the structure will lead to the change of the boundary of the flow field. In order to improve the efficiency of the calculation, the dynamic grid technique is used to update the mesh of the fluid domain. In this paper, the radial basis function interpolation method [9] is used to deform the mesh.

\subsection{Validation}

The numerical method described above is validated using the AGARD 445.6 wing [10]. This wing is an AGARD standard aeroelastic configuration with a NACA 65A004 airfoil section, a display length of $0.76 \mathrm{~m}$, a panel aspect ratio of 1.65 , a taper ratio of 0.66 and a $45^{\circ}$ quarter-chord sweep angle. The mesh number of wing surface in fluid and structural domain are 3693985 and 67507 respectively.

The structural material properties are set as $E_{1}=0.89 \mathrm{GPa}, E_{2}=1.54 \mathrm{GPa}, G=2.6 \mathrm{GPa}$ and $\rho=381.98 \mathrm{~kg} / \mathrm{m}^{3}$. Where $E_{1}$ and $E_{2}$ are elastic modulus of the wing along the chord and stretch. $G$, $v$ and $\rho$ are the shear modulus, Poisson's ratio and density respectively.

The computation conditions are $M a=0.8, \alpha=1^{\circ}, P=6412.685 \mathrm{~Pa}$ and $T=237.378 \mathrm{~K}$. After convergence, the deformation of the leading edge and trailing edge of the wings relative to the initial position are shown in Table 2. The method is close to Ref [10] and the error is less than 5\%, which shows that the numerical method in this paper is reliable.

Table 1. Comparison on results of static aeroelasticity of AGARD 455.6 wing

\begin{tabular}{|c|c|c|c|}
\hline Position & Paper & Reference & Error \\
\hline Leading edge $/(\mathrm{mm})$ & 10.667 & 11.176 & $4.55 \%$ \\
\hline Trailing edge $/(\mathrm{mm})$ & 13.050 & 12.700 & $2.76 \%$ \\
\hline
\end{tabular}

\subsection{Calculating model}

The model of the canard with forward-swept wing configuration is simplified in Ref. [2]. The geometry and geometric parameters of model are shown in Fig.1 and Table 2, respectively. The fuselage is formed as an elliptic cylinder with a canopy, with length $L_{F}$ of $2 \mathrm{~m}$, height $h_{F}$ of $0.25 \mathrm{~m}$, and width $w_{F}$ of $0.17 \mathrm{~m}$. 


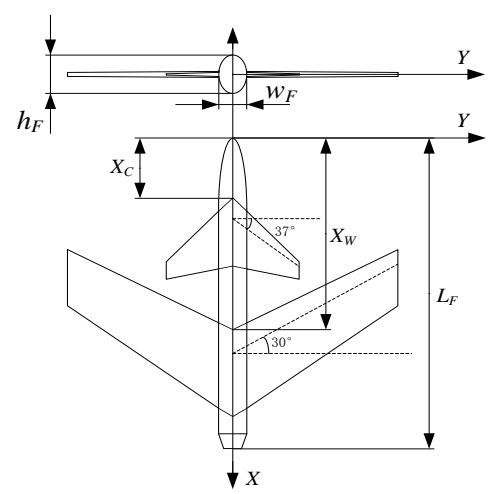

Fig.1 Outline of canard with forward-swept wing model

Table 2. Geometry dimension of wing and canard

\begin{tabular}{|c|c|c|c|c|c|}
\hline $\begin{array}{c}\text { Geometric } \\
\text { quantities }\end{array}$ & Span/(m) & $\begin{array}{c}\text { Aspect } \\
\text { ratio/(m) }\end{array}$ & $\begin{array}{c}\text { Root } \\
\text { chord/(m) }\end{array}$ & $\begin{array}{c}\text { Taper } \\
\text { ratio/(m) }\end{array}$ & Sweep/(m) \\
\hline Wing & 1.990 & 1.650 & 0.558 & 0.660 & $-30^{\circ}$ \\
\hline Canard & 0.800 & 1.160 & 0.445 & 0.250 & $37^{\circ}$ \\
\hline
\end{tabular}

The longitudinal positions of wing and canard are $X_{W}=0.622 L_{F}$ and $X_{C}=0.2 L_{F}$, and the vertical positions of wing and canard are in the horizontal symmetry of body.

\section{Results and analysis}

The aerodynamic characteristics and the bending-torsion deformation of canard-forward swept wing configuration are studied, which are compared with the forward swept wing configuration in this paper. The canard is rigid and the main wing have two different states (rigid and elastic) in the calculation. In order to simplify the following analysis, the canard-forward swept wing model and the forward swept wing model are respectively referred to as CW-FSW and FSW models.

The number of polyhedron mesh of CW-FSW and FSW model in fluid domain is 4.01 million and 4.8 million respectively, and the number of tetrahedron mesh in structural domain is 100,000 . The boundary conditions are $M a=0.5$ and $R e=4.8 \times 10^{6}$ in CFD solver. The boundary condition of the CSD solver is that the wing is fixedly connected with the fuselage, and the material properties are consistent with the above example.

\subsection{Aerodynamic characteristics}

The static aeroelastic characteristics of the CW-FSW and FSW models are calculated under the condition of high subsonic speeds. The results of the aerodynamic characteristics are shown in Fig.2. The rigid and elastic states in the following refer to the state of the wing. Wing root string at the leading edge point. The leading edge of the wing root is the reference point of the pitching moment.

The influence of the canard on the lift is divided into two aspects: the one is the lift producing by the canard itself, the other is the influence of canard on the wing. The influence of down wash of canard on the wing reduces the separation of the wing and increase the lift at large angle of attack, while reducing the lift at a small angle of attack.

According to Fig.3, when $\alpha<32^{\circ}$, the influence of down wash of canard on the wing causes the lift of the wing decrease and the unfavorable interference will be in domination. When $\alpha>32^{\circ}$, the vortex generated by the canard have favorable interference on the wing and can control the separation of the wing. The lift coefficient increases, but the increment is small. Meanwhile, the stall angle of attack of CW-FSW model will be delayed about $2^{\circ}$, which indicates that the canard can improve the maneuverability of the forward-swept wing.

Fig. 2(b) shows that the drag coefficient of CW-FSW model increases with the increasing of the lift coefficient, which indicates that the canard brings both the lift and the drag. Fig.2(c) displays the lift-drag ratio $\mathrm{K}$ of models. When $\alpha<8^{\circ}$, the $\mathrm{K}$ of the CW-FSW model is slightly lower than FSW 
model. When $\alpha>8^{\circ}$, the $\mathrm{K}$ of the CW-FSW model is slightly larger than FSW model, but the difference is not much.

From Fig. 2(d), the pitch moment coefficient of CW-FSW model is larger than that of FSW, because the position of canard is arranged before the torque reference point. At small angle of attack, same with the FSW model, the pitching moment of elastic CW-FSW is slightly larger than the rigid CW-FSW. When the angle of attack increase further, the pitching moment of the elastic CW-FSW decreases until smaller than the rigid one.

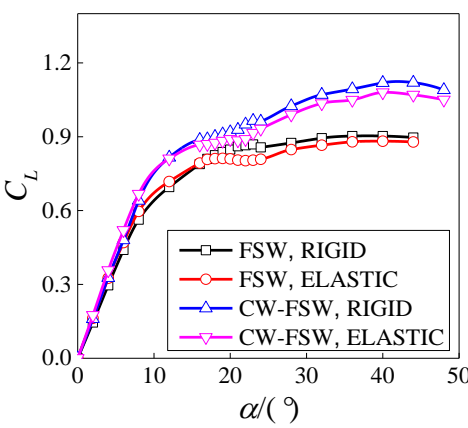

(a) Lift coefficient

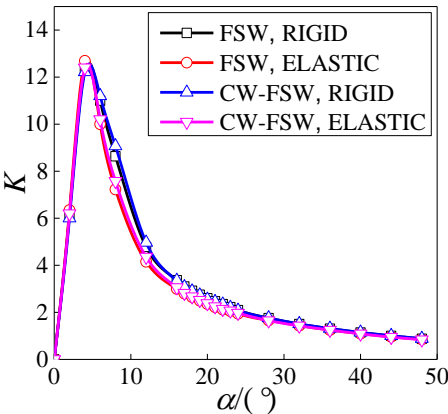

(c) Lift-drag ratio

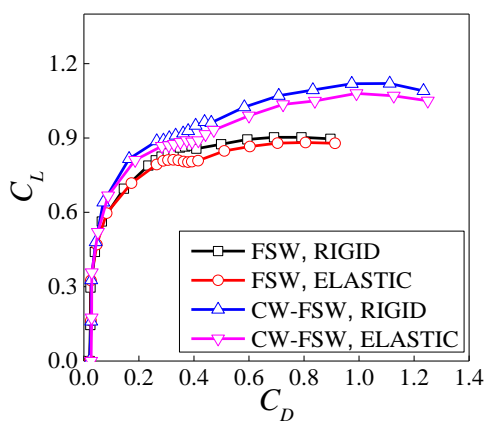

(b) Drag coefficient

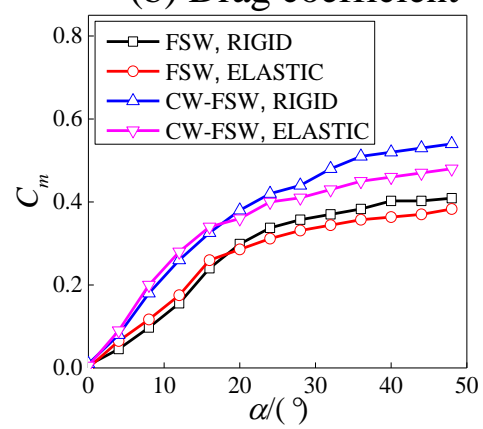

(d) Pitching moment coefficient

Fig. 2 Aerodynamic characteristics of model

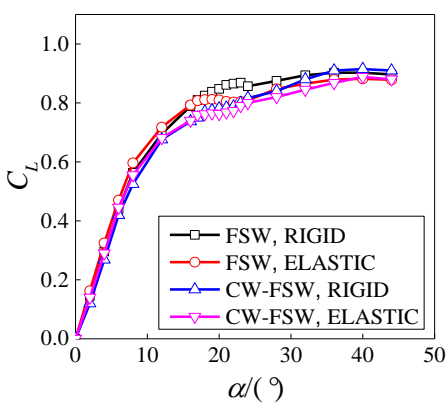

Fig.3 Lift coefficient of wing of model

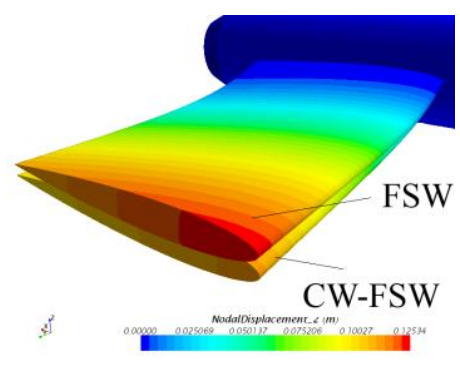

Fig. 4 Contours of displacement deformation

\subsection{Elastic deformation characteristics}

Figure 5 shows that the torsion and bending deformation of the wing of the FSW and CW-FSW models when $\alpha=4^{\circ}$ and $\alpha=8^{\circ}$.It can be seen from Fig. 5(a) that the twist angle of the wing of the CW-FSW model is larger than that of FSW, while the leading edge displacement of the CW-FSW wing is slightly smaller than that of the FSW wing. Figure 4 and 5(b) give out the contours of displacement deformation of the wing of FSW and CW-FSW models, which indicates that the deflection of CW-FSW wing is significantly smaller than that of FSW.

Figure 6(a) shows the variation of twist angle with the angle of attack of wing of FSW and CW-FSW model, and figure 6 (b) displays the variation of displacement in the direction of $\mathrm{Z}$ axis of leading edge point with the angle of attack. 
As shown in Fig. 6(a), the trend of twist angle of wingtip $\lambda$ changing with the angle of attack $\alpha$ for CW-FSW are basically same as FSW. When $\alpha<8^{\circ}$, the twist angle of the CW-FSW wing is slightly larger than FSW. When $\alpha>8^{\circ}$, the reduction of the CW-FSW twist angle is more sharp. Increasing with the angle of attack, the reduction stays at about $1.8^{\circ}$. Seen from Fig. 6(b), at moderate angle of attack, compared with the FSW, the deflection of CW-FSW wing reduces obviously. In the vicinity of $\alpha=20^{\circ}$, the deflection of the CW-FSW wing can't produce a sudden drop but a softer shortfall. Indicating that the canard can delay the wing stall and lead lift coefficient of the wing to change gently around the stall angle of attack.

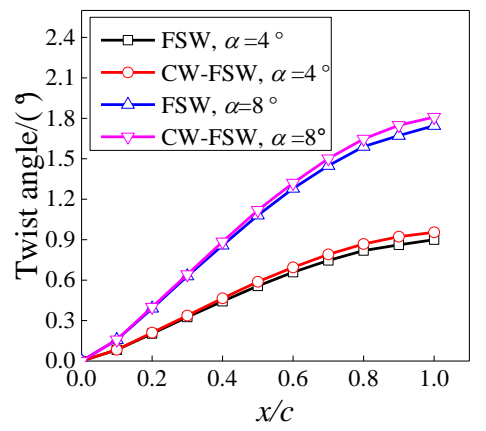

(a) Torsion deformation

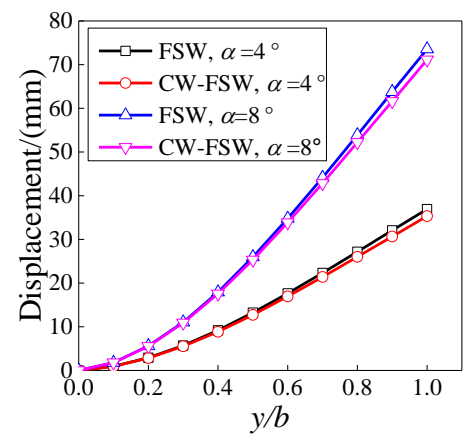

(b) Bending deformation

Fig. 5 Bending and torsion deformation of wing

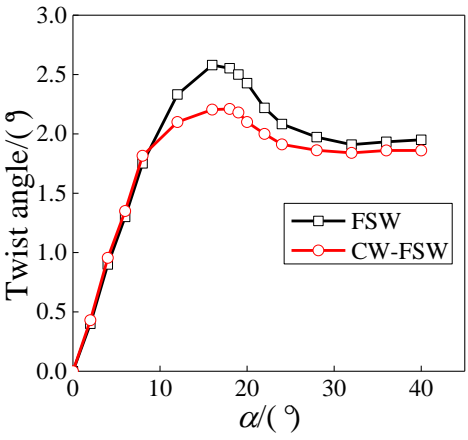

(a) Torsion deformation

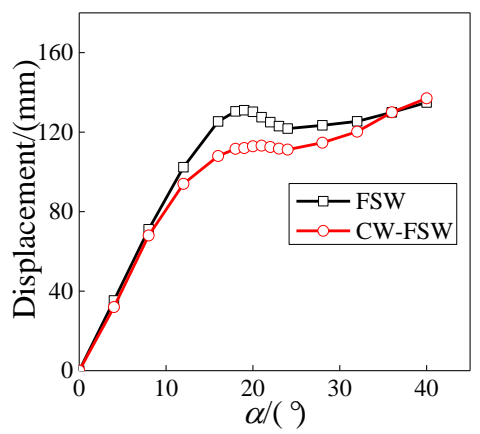

(b) Bending deformation

Fig. 6 Relationship between angle of attack and deformation of wing

\subsection{Flow mechanism analysis}

The pressure coefficient of wing on different positions is shown in Fig.7. The peak of pressure on the surface of the wing of FSW model is much larger than that of the CW-FSW on position $y / b=25 \%$ near the root of wing. In the middle of the wing at $y / b=45 \%$, the peak of pressure on the surface of the wing of FSW model is smaller than CW-FSW. And the pressure coefficient is almost the same at the near wingtip $\mathrm{y} / \mathrm{b}=80 \%$, which indicates that the canard have little interference with airflow at the wingtip.

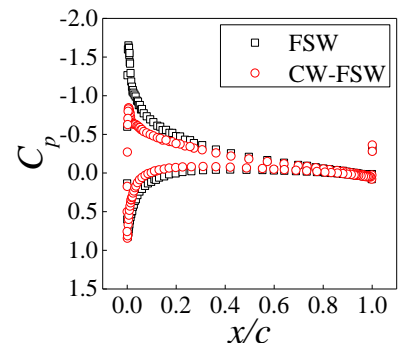

(a) $y / b=25 \%$

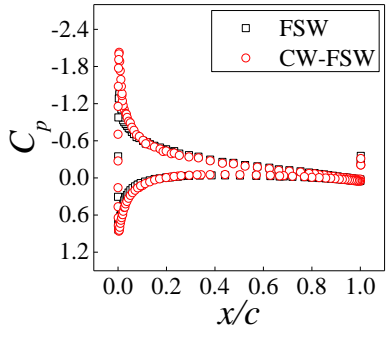

(b) $y / b=45 \%$

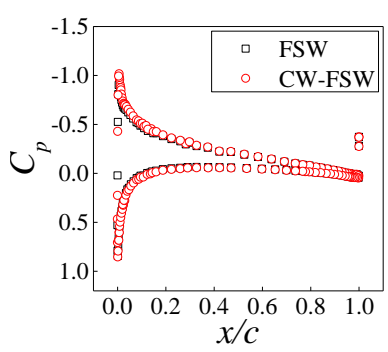

(c) $\mathrm{y} / \mathrm{b}=80 \%$

Fig. 7 Pressure coefficient on different position when $\alpha=4^{\circ}$

As shown in Fig.8, the minimum negative pressure area of the upper surface of wing is transferred from the wing root to the middle of the wing under the effect of the canard, so the bending and torsion deformation of the wing of CW-FSW is slightly more than FSW. However, the deformation is very small, so the change of aerodynamic characteristics of flexible wing caused by canard is very little under the conditions of the small angle of attack. 


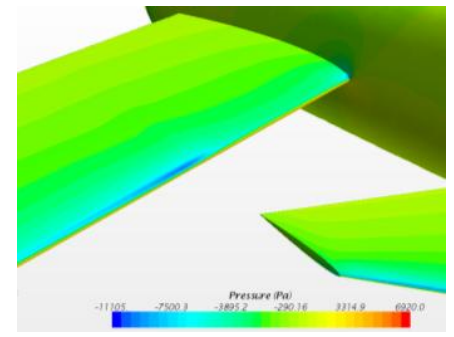

(a) CW-FSW model

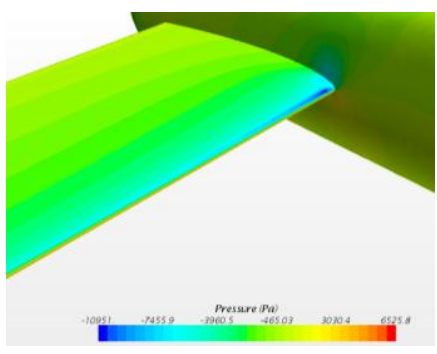

(b) FSW model

Fig. 8 Pressure contours of leading edge of wing

Figure 9 displays the contours of pressure and spatial streamlines for FSW and CW-FSW. As shown in Fig.9, the vortex produced by the canard has obvious extrapolation effect on the vortex produced by the wing, which reduces the negative pressure area of the wing upper wing and the lift of the wing and restrains the elastic deformation of the wing.

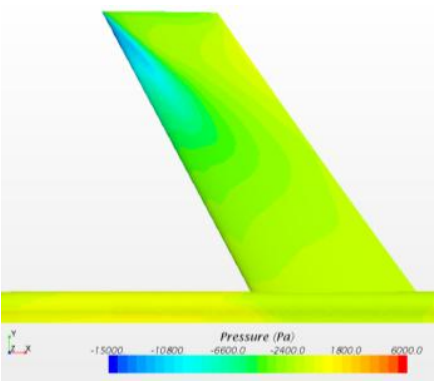

(a) Pressure contours of FSW

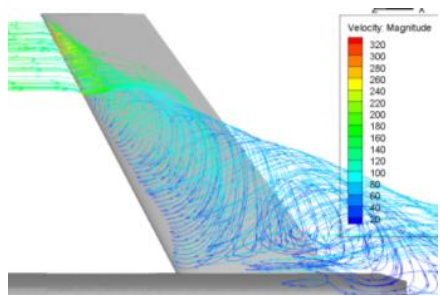

(c) Streamlines of FSW

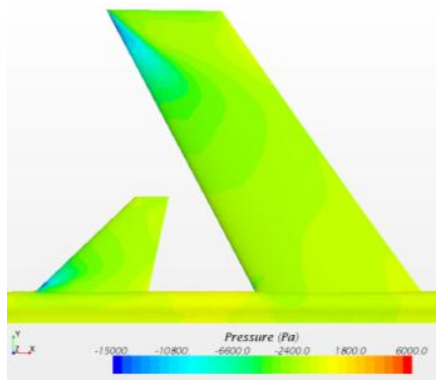

(b) Pressure contours of CW-FSW

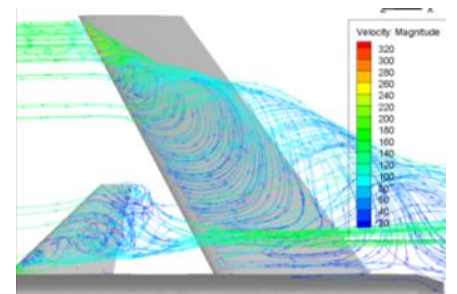

(d) Streamlines of CW-FSW

Fig. 9 Pressure contours and streamlines of model when $\alpha=16^{\circ}$

The streamlines of FSW and CW-FSW at the angle of attack $20^{\circ} \sim 24^{\circ}$ are shown in Figure 10 . When $\alpha=20^{\circ}$, The nucleus of vortex of FSW has ruptured and the wing stalls while the vortex of surface of CW-FSW is very stable. For CW-FSW model, because the vortex of canard and the leading edge vortex of wing rotate in the opposite direction, resulting in favorable interference, energy enhanced each other, so the flow on outside is more stable, and breakdown of vortex is delayed.

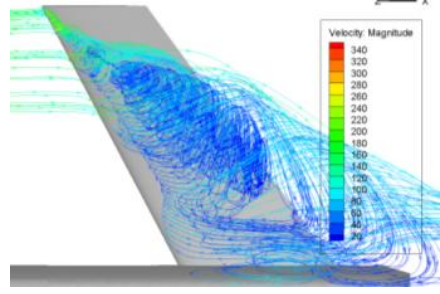

(a) FSW, $\alpha=20^{\circ}$

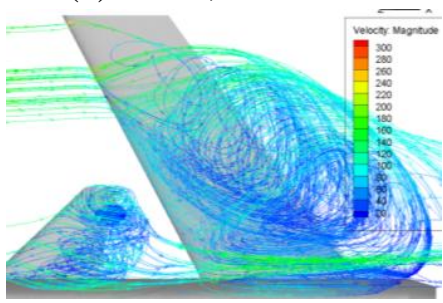

(c) CW-FSW, $\alpha=22^{\circ}$

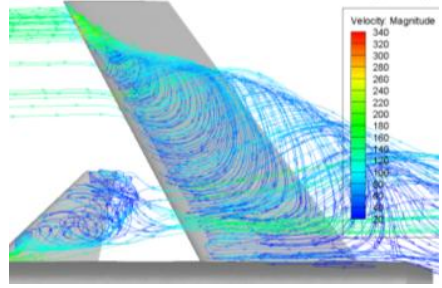

(b) CW-FSW, $\alpha=20^{\circ}$

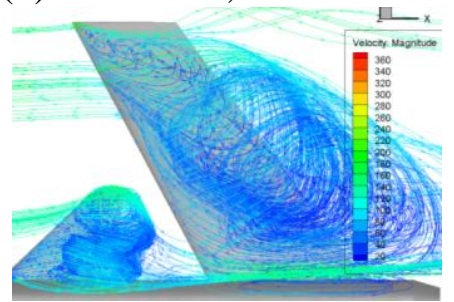

(d) CW-FSW, $\alpha=24^{\circ}$

Fig. 10 Pressure contours and streamlines of model when $\alpha=20^{\circ} \sim 24^{\circ}$ 
When the angle of attack is further increased to $22^{\circ}$, the surface of CW-FSW wing produce vortex core, and lift coefficient reaches the maximum. When $\alpha=24^{\circ}$, the nuclear of vortex breakdown, and the wing stall.

\section{Conclusion}

Aiming at the influence of canard on the aerodynamic characteristics and elastic deformation characteristics of the elastic forward swept wing, calculation and analysis of the static aero-elastic characteristics of the canard with forward swept wing configuration based on the improved CFD / CSD loosely coupled numerical method is carried out, and compared with the forward swept wing configuration, the conclusions are as follows:

(1) CFD / CSD loosely coupled numerical method is more accurate and can be used in the analysis of static aroelasticity of canard-forward swept wing configuration.

(2) The aerodynamic characteristics of the wing in the elastic state and rigid state are similar under the influence of the canard. When $\alpha<32^{\circ}$, the adverse effects of down wash of canard on the wing play a leading role, and the lift coefficient decreased. When $\alpha>32^{\circ}$, the vortex produced by canard can control the flow separation of wing, which produce favorable interference, and lead to the increase of lift coefficient, but the increment is small.

(3) The bending and torsion deformation of the forward swept wing under the influence of the canard wing is obviously restrained, which is beneficial to improve the stability of static aeroelasticity of the forward swept wing aircraft.

\section{References}

[1]. Fang Bao-rui. Aircraft aerodynamic configuration design. Beijing: Aviation Industry Press, 1997.

[2]. Breitsamter C, Laschka B. Vortical flowfield structure at forward swept-wing configurations. Journal of Aircraft, 2001, 38(2): 193-207.

[3]. Wang Jin-jun, Zhao Xia, Wang Shuangfeng, et al. Experimental investigation on longitudinal aerodynamic characteristics of Canard-Forward-Swept wing configuration. Acta Aerodynamica Sinica, 2004; 22(2): 237-240.

[4]. Ren Zhi-jing, Wang Xu, Liu Wen-fa. Numerical Simulation on Aerodynamic Influence of Canard on Forward-swept Configuration. Acta Aeronautica et Astronautica Sinica, 2010; 31(7): 1318-1323.

[5]. Wang Yi-kun, Shi Ai-ming. Static aeroelastic correction for wind tunnel results of wing model with CFD. Science Technology and Engineering, 2012; 12(15): 3685-3688.

[6]. Chen Da-wei, Yang Guo-wei. Static aeroelastic analysis of a flying-wing using different models. Chinese Journal of Theoretical and Applied Mechanics, 2009; 41(4): 469-479.

[7]. Feng Hao-yang, Su Xin-bing, Ma Bin-lin, et al. Static Aero-elastic Computation with Polyhedron Grid. Flight Dynamics， 2016; 34(4):24-28.

[8]. Xu Ming, Chen Shi-lu. Study of data exchange method for coupling computational CFD/CSD. Chinese Journal of Applied mechanics, 2004; 21(2): 33-36.

[9]. Wang Gang, Lei Bo-qi, Ye Zheng-yin. An efficient deformation technique for hybrid unstructured grid using radial basis functions. Journal of Northwestern Polytechnical University, 2011; 29(5): 783-788.

[10]. Goura G S L. Time marching analysis of flutter using computational fluid dynamics. University of Glasgow, 2001. 